\title{
Geoeffectiveness and efficiency of CIR, Sheath and ICME in generation of magnetic storms
}

Yu. I. Yermolaev, ${ }^{1}$ N. S. Nikolaeva ${ }^{1}$ I. G. Lodkina, ${ }^{1}$ M. Yu. Yermolaev ${ }^{1}$

\footnotetext{
${ }^{1}$ Space Plasma Physics Department, Space Research Institute, Russian Academy of Sciences, Profsoyuznaya 84/32, Moscow

117997, Russia. (yermol@iki.rssi.ru)
} 


\section{Abstract.}

We investigate relative role of various types of solar wind streams in generation of magnetic storms. On the basis of the OMNI data of interplanetary measurements for the period of 1976-2000 we analyze 798 geomagnetic storms with $D s t \leq-50 \mathrm{nT}$ and their interplanetary sources: corotating interaction regions (CIR), interplanetary CME (ICME) including magnetic clouds (MC) and Ejecta and compression regions Sheath before both types of ICME. For various types of solar wind we study following relative characteristics: occurrence rate; mass, momentum, energy and magnetic fluxes; probability of generation of magnetic storm (geoeffectiveness) and efficiency of process of this generation. Obtained results show that despite magnetic clouds have lower occurrence rate and lower efficiency than CIR and Sheath they play an essential role in generation of magnetic storms due to higher geoeffectiveness of storm generation (i.e higher probability to contain large and longterm southward IMF $B z$ component). 


\section{Introduction}

One of key issues of the solar-terrestrial physics is investigation of mechanisms of energy transfer from the solar wind into the magnetosphere and of excitation of magnetospheric disturbances. As has been discovered by direct space experiments in the beginning of 1970s, the basic parameter leading to magnetospheric disturbances is negative (southward) Bz component of interplanetary magnetic field (IMF) (or electric field $E y=V x \times B z$ ) [Dungey, 1961; Fairfield and Cahill, 1966; Rostoker and Falthammar, 1967; Russell et al., 1974; Burton et al., 1975; Akasofu, 1981]. Numerous investigations demonstrated that IMF in the undisturbed solar wind lies in the ecliptic plane (i.e., Bz is close to zero) and only disturbed types of the solar wind streams can have a considerable value of IMF Bz. The interplanetary CME (ICME) with a compression region Sheath before it and the compression region between slow and fast solar wind streams (Corotating Interaction Region, CIR) belong to such types of solar wind streams (see reviews and recent papers, for instance, by Tsurutani et al. [1988]; Tsurutani and Gonzalez [1997]; Gonzalez et al. [1999]; Yermolaev and Yermolaev [2002]; Huttunen and Koskinen [2004]; Echer and Gonzalez [2004]; Yermolaev and Yermolaev [2006]; Borovsky and Denton [2006]; Denton et al. [2006]; Huttunen et al. [2006]; Yermolaev et al. [2007a, b, c]; Pulkkinen et al. [2007a, b];

Zhang et al. [2007]; Turner et al. [2009]; Yermolaev et al. [2010a, b, c, d, 2011]; Nikolaeva et al. [2011]; Alves et al. [2011]; Echer et al. [2011]; Gonzalez et al. [2011]; Guo et al. [2011]; Mustajab and Badruddin [2011] and references therein).

Experimental results showed that the magnetospheric activity induced by different types of interplanetary streams is different [Borovsky and Denton, 2006; Denton et al., 2006; 
Huttunen et al., 2006; Pulkkinen et al., 2007a; Plotnikov and Barkova, 2007; Longden et al., 2008; Turner et al., 2009; Despirak et al., 2009, 2011; Guo et al., 2011]. This fact indicates that it is necessary to take into account the influence of other (in addition to IMF $B z$ and electric field $E y)$ parameters of the solar wind, dynamics of parameter variation, and different mechanisms of generating the magnetospheric disturbances at different types of solar wind streams. Several recent papers analyzed separately CIR, Sheath and body of ICME and compared them with each other [Huttunen and Koskinen, 2004; Yermolaev and Yermolaev, 2006; Huttunen et al., 2006; Yermolaev et al., 2007a, b, c; Pulkkinen et al., 2007a; Yermolaev and Yermolaev, 2010; Yermolaev et al., 2010a, b, 2011; Alves et al., 2011; Despirak et al., 2011; Nikolaeva et al., 2011; Guo et al., 2011].

Papers mentioned above are devoted studying of response of magnetosphere to interplanetary drives and use word geoeffectiveness for a designation of this link. It should be noted that there is a double meaning of the term geoeffectiveness. In one case, geoeffectiveness implies a probability with which selected phenomenon can cause a magnetic storm, i.e., the ratio between the number of events of a chosen type resulting in a magnetic storm and the total number of these events. In the other case, geoeffectiveness implies the efficiency of storm generation by unambiguously interrelated phenomena, i.e., the ratio between the "output" and "input" of a physical process, for example, between the values of the Dst index and the southward IMF Bz component. Below we will use the term geoeffectiveness for a designation of probability of relation between the phenomena and the term efficiency for a designation of efficiency of process relating phenomena.

A considerable quantity of papers is devoted investigations of geoeffectiveness in generation of magnetic storm. The great bulk of works studies geoeffectiveness of magnetic 
clouds, and geoeffectiveness of other phenomena is studied rather poorly (see, for example, one of recent reviews by Yermolaev and Yermolaev [2006, 2010]; Alves et al. [2011]. So, one of the main aims of current paper is investigation of geoeffectiveness of various interplanetary drivers and comparison of them to each other.

Efficiencies of various interplanetary drivers vary with the type of solar wind streams and may be estimated as the ratio of measured energy output to estimated energy input (see, for example, paper by Turner et al. [2009]; Yermolaev et al. [2010c] and references therein). In our investigations, we use Bz (Ey) and magnetospheric indices Dst, Dst* (pressure corrected $D s t$ ), $K p$ and $A E$ as "input" and "output" of the storm generation processes for the estimation of efficiency of interplanetary drivers.

\section{Methods}

When the types of solar wind streams were classified, we used OMNI database (see http://omniweb.gsfc. nasa.gov [King and Papitashvili, 2004]) for interval 1976-2000 and available world experience in identification of solar wind streams and the standard criteria for following parameters: velocity $V$, density $N$, proton temperature $T$, ratio of thermal to magnetic pressure ( $\beta$-parameter), ratio of measured temperature to temperature calculated on basis of average "velocity-temperature" relation T/Texp [Lopez, 1987], thermal pressure and magnetic field. This method allows us to identify reliably 3 types of quasi-stationary streams of the solar wind (heliospheric current sheet (HCS), fast streams from the coronal holes, and slow streams from the coronal streamers), and 5 disturbed types (compression regions before fast streams (CIR), and interplanetary manifestations

of coronal mass ejections (ICME) that can include magnetic clouds (MC) and Ejecta with the compression region Sheath preceding them). In contract with Ejecta, MCs have 
lower temperature, lower ratio of thermal to magnetic pressure ( $\beta$-parameter) and higher, smooth and rotating magnetic field [Burlaga, 1991]. In addition, we have included into our catalog such events (rare enough) as direct and reverse shocks, and the rarefaction region (region with low density) Rare but these types of events are not analyzed in this paper.

In order to calculate yearly averaged values, we have taken into consideration that the OMNI database contains gaps of the data from 0 to $50 \%$ time of year. This procedure has been made in the assumption that occurrence rate of given type of solar wind streams is similar both in intervals of data presence and in intervals of data gap. If during chosen year the number of events of selected solar wind type $N e$ has been registered in interval of data presence $t_{d}$ the normalized number of the given solar wind type $N e^{*}$ in this year was defined by multiplication of occurrence rate of the given solar wind type $N e / t_{d}$ to total duration of year $t_{y}$, i.e. $N e^{*}=\left(N e / t_{d}\right) * t_{y}$. Normalized number of solar wind events is used only for studying the time variations in occurrence rate of various types of streams and measured number of events is used to calculate geoeffectiveness of types of events. When we analyzed durations of different types of solar wind streams, we selected intervals of types of streams which have not data gaps at both edges of the intervals.

Definite types of the solar wind streams were put in correspondence to all magnetic storms for which measurements of the parameters of plasma and magnetic field in the interplanetary medium were available. This was done using the following algorithm. If the moment of minimum in the $D s t$ index from the list of magnetic storms falls within the time interval of a solar wind event or is apart from it by no more than $2 \mathrm{~h}$ interval, the corresponding solar wind type is ascribed to this storm. It should be noted that, 
according to the results of analysis of 64 intense $(D s t<-85 \mathrm{nT})$ magnetic storms in the period 1997-2002, the average time delay between Dst peak and southward IMF Bz component is equal to $\sim 2 \mathrm{~h}$ [Gonzalez and Echer, 2005]. Similar results were obtained in papers by Yermolaev et al. [2007a, c]. Thus, two hours correspond to the average time delay between the Dst peak of an intense magnetic storm and the associated peak in the southward IMF Bz component.

In order to investigate the dynamic relation between development of parameters in interplanetary sources and in the magnetospheric indices we apply the method of double superposed epoch analysis (DSEA) [Yermolaev et al., 2010c, d]. Two reference times are used in this method: we put together the time of storm onset (time "0") and time of Dst index minimum (time "6"), the data between them we compress or expand in such a way that durations of the main phases of all magnetic storms is equal to each other. This DSEA method allows us to simultaneously study interplanetary conditions resulting in the beginning and end of magnetic storms as well as dynamics (temporal variations) of parameters during main phase for storms with different durations.

\section{Results}

Obtained results are presented in 3 subsections devoted to ( 1) observational statistics of various types of solar wind streams, (2) probability of magnetic storm generation by these interplanetary drivers, and (3) efficiency of magnetic storm generation by various drivers. 


\subsection{Occurrence rate of different types of solar wind streams}

In order to estimate geoeffectiveness of different types of solar wind streams it is necessary to have a total list of these types of streams during sufficiently large time interval and with sufficiently large statistics. Measured and normalized numbers per year, average durations, temporal parts in total times of observations as well as average values and their standard deviations of several plasma and magnetic field parameters for various solar wind types have been presented in our publication [Yermolaev et al., 2009, 2010a, b, c, d, 2011]. It should be noted that both types of compressed regions (CIR and Sheath) have very close values of parameters while the parameters for 2 types of ICME (Ejecta and MC) are different. In figure 1 we present yearly average values of sunspots (top panel) and yearly average distributions of times of observations for different types of solar wind streams (bottom panel). Data for different types of streams are showed by various color columns (see designation on the right of the figure) with height proportional to percent of observation time. On the average the quasi-steady types of solar wind streams (Fast, Slow and HCS) contain about $60 \%$ of all solar wind observations near the Earth but time of disturbed types of streams decreases down to $25 \%$ during solar minimum and increases up to $50 \%$ during solar maximum.

Various types of solar wind streams transport different values of mass, momentum, energy and magnetic field from the Sun to the Earth. Figure 2 shows average distributions (percentage) of values (red columns) and total Sun's losses (parameters integrated over time, blue columns) mass, momentum, energy and magnetic fluxes for different types of solar wind streams. High average values for mass, momentum, and energy fluxes are observed in compressed regions CIR and Sheath and magnetic flux in MC, but their total 
losses are higher in steady types of streams (Fast and Slow) than in disturbed types of streams. In following sections of the paper we will analyze how occurrence rate of different types of streams and mass, momentum, energy and magnetic field transferred by these streams influence generation of magnetic storms.

\subsection{Geoeffectiveness of interplanetary drivers}

For the entire period of time 1976-2000, 798 moderate and strong magnetic storms with the intensity $D s t \leq-50 \mathrm{nT}$ were observed on the Earth (see figure 3). But only for 464 magnetic storms (i.e., for $58 \%$ of all magnetic storms) corresponding various events were found in the solar wind. The sources of other 334 magnetic storms (i.e., of $42 \%$ of 798 storms, grey columns in figure 3) are undeterminate, and this fact is mainly connected with the lack of data on plasma and interplanetary magnetic field which makes impossible to identify the solar wind type for magnetic storm intervals. Figure 4 presents distribution of storms when we excluded IND storms from analyses.

Analyses of data in Figures 1 and 4 allows us to compare number of each type of solar wind streams and number of magnetic storms induced by these types of streams and to calculate probability (geoeffectiveness) of generation of magnetic storms by each types of these interplanetary drivers (see Table 2). Though the statistics of annual numbers of solar wind streams is small, the available data speak in favour of suggestion that geoeffectiveness does not change essentially during solar cycle.

\subsection{Efficiency of interplanetary drivers}

One of important problems of connection between interplanetary conditions and magnetospheric processes is the dependence of magnetospheric activity on temporal evolution 
of solar wind plasma and IMF parameters including Bz and Ey. We found a consistency between time evolution of cause ( $\mathrm{Bz}$ and $\mathrm{Ey})$ and time evolution of effect (Dst, Dst* (pressure corrected Dst), $K p$ and $A E$ indices) for the time interval of "0"-"6" as dependence of indices on integral value of sources, for example, $D s t^{i} \cdot v s . E y\left(\sum\right)^{i}=\int_{0}^{t^{i}} E y(\tau) d \tau=$ $\sum_{0}^{i} E y^{k}, i=0, \ldots, 6 ; k=0, \ldots, i$.

Dependencies of $D s t$ (or $D s t^{*}$ ) on the integral of Bz (or Ey) over time are almost linear and parallel for different types of drivers. This fact can be considered as an indication that time evolution of main phase of storms depends not only on current values of $\mathrm{Bz}$ and Ey, but also on their prehistory. The differences between these lines are relatively small $(|\Delta D s t|<20 \mathrm{nT})$. Nevertheless we can make following comparisons. For various drivers we approximated data near central parts dependencies by linear functions and calculated values of $D s t$ (or $D s t^{*}$ ) at fixed values of integral of $B z$ and integral of Ey $\left(\int_{0}^{t} B z(\tau) d \tau=-30 \mathrm{~h}^{*} \mathrm{nT}\right.$ and $\left.\int_{0}^{t} E y(\tau) d \tau=12 \mathrm{~h}^{*} \mathrm{mV} / \mathrm{m}\right)$ (see Table 3$)$. It should be noted that used value of integral of $E y$ is located near threshold of generation of magnetic storms with $D s t \leq-50$ nT (i.e. used interval of integral of Ey contains data for almost all magnetic storms) [Nikolaeva et al., 2012]. Taking into account that difference in "efficiency coefficients" for various drivers are mathematically significant when they differ more than $10 \%$ (i.e. $0.25 \mathrm{nT} /\left(\mathrm{h}^{*} \mathrm{nT}\right)$ for $B z$ and $0.5 \mathrm{nT} /\left(\mathrm{h}^{*} \mathrm{mV} / \mathrm{m}\right)$ for $\left.E y\right)$, it is possible to note that: (1) Dependencies of Dst (or Dst $t^{*}$ on the integral of Bz (or Ey) are higher in CIR, Sheath and Ejecta, than in MC, (i.e., efficiency of MC for process of magnetic storm generation is the lowest one); (2) Efficiency of CIR, Sheath and Ejecta are closed to each other. Dependencies of $K p$ (and $A E$ ) on integral of $\mathrm{Bz}$ (and Ey) are nonlinear (there is the saturation effect for $A E$ index) and nonparallel. Nevertheless we made the same 
procedure for them as for Dst and $D s t^{*}$ indices and calculated estimations of efficiency for different drivers. Efficiency for $K p$ and $A E$ indices is higher for CIR and Sheath than for $\mathrm{MC}$ and Ejecta.

\section{Discussion and conclusions}

The quantity of Sun's energy flowing in a magnetosphere and causing magnetospheric disturbances, is defined by following processes and relations:

1. relative occurrence rate of disturbed types of solar wind streams (interplanetary drivers of magnetic storms),

2. typical values of plasma and field parameters in these types of streams,

3. probability in magnetic storm generation (geoeffectiveness) for these drivers (i.e. probability of occurrence of southward IMF Bz component in these drivers), and

4. efficiency of physical process of magnetic storm generation for various drives.

On the basis of OMNI data during 1976-2000 we estimated and compared these processes and relations.

The results of our identification of solar wind streams were partially compared with tabulated data of various events presented on the websites http://star.mpae.gwdg.de/, http://lempfi.gsfc.nasa.gov/, and with the ISTP Solar Wind Catalog on the website http://www-spof.gsfc.nasa.gov/scripts/sw-cat/Catalog- events.html. and presented in papers by Cane and Richardson [2003]; Alves et al. [2006]; Koskinen and Huttunen [2006]; Echer et al. [2006]; Zhang et al. [2007]. This comparison showed a good agreement in more than $90 \%$ of events. It is important to note that, unlike numerous papers where solar wind identifications were made for selection of only one or two stream types we realized this approach with a single set of criteria to eight large-scale stream types. The obtained sta- 
tistical characteristics and distributions of the solar wind and IMF parameters in various types of the streams well agree with previously obtained results

During the full time from 1976 to 2000 the different types of the solar wind were observed: HCS for $6 \pm 4 \%, \mathrm{MC}$ for $2 \pm 1 \%$, Ejecta for $20 \pm 6 \%$, Sheath before Ejecta for $8 \pm 4 \%$, Sheath before MC for $0.8 \pm 0.7 \%$, and CIR for $10 \pm 3 \%$ of the total observation time. About $53 \%$ of the entire observation time fell on fast and slow solar wind (21.5\% and $31.5 \%$ of time, respectively) (see Figure 1 and Table 1). Large values of mass, momentum and energy are transported from the Sun to the Earth by CIR and Sheath, and of magnetic field by MC (see Figure 2).

Probabilities that conditions in the interplanetary space allow to input solar wind energy to magnetosphere and generate magnetic storm with $D s t \leq-50 \mathrm{nT}$ are about $55 \%$ for $\mathrm{MC}$ (63\% for MC with Sheath), about $20 \%$ for CIR, about $8 \%$ for Ejecta ( $21 \%$ for Ejecta with Sheath) and $15 \%$ for Sheath (see Table 2). Because of different occurrence rates of different solar wind streams it was found that $35 \%$ storms were generated by Ejecta with/without Sheath, $31 \%$ by CIR and $24 \%$ by MC with/without Sheath (about $20 \%$ by Sheath before MC and Ejecta). Taking into account dependence of numerical estimation on used method of data analysis, values of geoeffectiveness obtained by us for MC and Ejecta (both with Sheath and without Sheath) are in a good agreement with previous result (see review by Yermolaev and Yermolaev [2010]). Our estimation of CIR geoeffectiveness (about 20\%) is lower than one obtained early by Alves et al. [2006].

Our estimations show that efficiency of MC for process of magnetic storm generation (for Dst and $D s t^{*}$ indices) is the lowest one and efficiency for $K p$ and $A E$ indices is higher for CIR and Sheath than for MC and Ejecta. Higher efficiency of magnetic storms 
generation process by Sheath than MC are discussed in several papers [Huttunen and Koskinen, 2004; Huttunen et al., 2006; Yermolaev et al., 2007a, b, c, 2010c; Pulkkinen et al., 2007a; Turner et al., 2009; Guo et al., 2011]. Our results confirm this conclusion.

Thus obtained results show that despite low occurrence rate and low efficiency of magnetic clouds they play an essential role in generation of magnetic storms due to high geoeffectiveness of storm generation (i.e high probability to contain large and long-term southward IMF $B z$ component). Geoeffectivenesses of CIR and Sheath are lower but they are compensated by higher occurrence rate and efficiency.

Acknowledgments. The authors are grateful for the possibility of using the OMNI database. The OMNI data were obtained from the GSFC/SPDF OMNIWeb on the site http://omniweb.gsfc.nasa.gov. This work was supported by the Russian Foundation for Basic Research, projects nos. 07-02-00042 and 10-02-00277a, and by the Program 16 of Physics Department of Russian Academy of Sciences (OFN RAN).

\section{References}

Akasofu, S.-I. (1981), Energy coupling between the solar wind and the magnetosphere, Space Sci. Rev., 111, A07S08, doi:10.1029/2005JA011447.

Alves, M. V., Echer, E., and Gonsalez, W. D., (2006) Geoeffectiveness of Corotating Interaction Regions as Measured by Dst Index, J. Geophys. Res., 2006, vol. 111, p. A07S05. doi: 10.1029/2005JA011379.

Alves, M. V., E.Echer, W.D.Gonzalez, (2011), Geoeffectiveness of solar wind interplanetary magnetic structures, Journal of Atmospheric and Solar-Terrestrial Physics, 73, $1380-1384$. 
Borovsky, J. E. and Denton, M.H. (2006), Differences between CME-Driven Storms and CIR-Driven Storms, J. Geophys. Res., 28, 121-190.

Burlaga, L. F. (1991), Magnetic clouds, in Physics of the Inner Heliosphere, edited by R. Schwenn and E. Marsch, Springer-Verlag, New York, V.2. P.1.

Burton, R. K., McPherron, R. L., and Russell, C. T (1975), An empirical relationship between interplanetary conditions and Dst, J. Geophys. Res., 80, 4204-4214.

Cane, H. V. and Richardson, I.G., (2003), Interplanetary Coronal Mass Ejections in the Near-Earth Solar Wind during 1996-2002, J. Geophys. Res., 2003, vol. 108, no. A4. doi: 10.1029/2002JA009817.

Denton, M. H., Borovsky, J. E., Skoug, R. M., Thomsen, M. F., Lavraud, B., Henderson, M. G., McPherron, R. L., Zhang, J. C., and Liemohn, M. W. (2006), Geomagnetic storms driven by ICME and CIR-dominated solar wind, J. Geophys. Res., 111, A07S07, doi:10.1029/2005JA011436.

Despirak, I. V., Lubchich, A.A., Yahnin, A.G., et al. (2009), Development of Substorm Bulges during Different Solar Wind Structures, Ann. Geophys., vol. 27, no. 5, pp. 19511960.

Despirak, I. V., A.A.Lubchich, V.Guineva (2011), Development of substorm bulges during storms of different interplanetary origins, Journal of Atmospheric and Solar-Terrestrial Physics, 73, 1460-1464.

Dungey, J. W. (1961), Interplanetary Magnetic Field and the Auroral Zones, Phys. Rev. Lett., 6, 47-48.

Echer, E. and Gonzalez, W. D. (2004), Geoeffectiveness of interplanetary shocks, magnetic clouds, sector boundary crossings and their combined occurrence, Geophys. Res. Lett., 
31, L09808, doi:10.1029/2003GL019199.

Echer, E., Gonsalez, W.D.., and Alves, M.V., (2006), On the Geomagnetic Effects of Solar Wind Interplanetary Magnetic Structures, Space Weather, 2006, vol. 4, p. S06001. doi: 10.1029/2005SW000200.

Echer, E., W. D. Gonzalez, B. T. Tsurutani, (2011), Statistical studies of geomagnetic storms with peak Dst $<-50$ nT from 1957 to 2008 Journal of Atmospheric and SolarTerrestrial Physics, 73, 1454-1459.

Fairfield, D. H. and Cahill, Jr., L. J. (1966), The transition region magnetic field and polar magnetic disturbances, J. Geophys. Res., 71, pp.155-169.

Gonzalez, W. D., Tsurutani, B. T., and Clua de Gonzalez, A. L. (1999), Interplanetary origion of geomagnetic storms, Space Sci. Rev., 88, 529-562.

Gonzalez, W.D., and Echer, E., A (2005), Study on the Peak Dst and Peak Negative Bz Relationship during Intense Geomagnetic Storms, Geophys. Res. Lett., vol. 32, L18103. doi: 10.1029/2005GL023486.

Gonzalez W. D., E. Echer, B. T. Tsurutani, A. L. Clua de Gonzalez, A, Dal Lago, (2011), Interplanetary Origin of Intense, Superintense and Extreme Geomagnetic Storms, Space Sci Rev, 158, 69-89. DOI 10.1007/s11214-010-9715-2

Guo, J., X. Feng, B. A. Emery, J. Zhang, C. Xiang, F. Shen, and W. Song (2011), Energy transfer during intense geomagnetic storms driven by interplanetary coronal mass ejections and their sheath regions, J. Geophys. Res., 116, A05106, doi:10.1029/2011JA016490

Huttunen, K. E. J. and Koskinen, H. E. J. (2004), Importance of postshock streams and sheath region as drivers of intense magnetospheric storms and high-latitude activity, 
Ann. Geophys., 22, 1729-1738, doi:10.5194/angeo-22-1729-2004.

Huttunen, K.E.J., Koskinen, H.E.J., Karinen, A., and Mursula, K. (2006), Asymmetric Development of Magnetospheric Storms during Magnetic Clouds and Sheath Regions, Geophys. Res. Lett., vol. 33, p. L06107.doi: 10.1029/2005GL024894.

King, J.H. and Papitashvili, N.E., (2004), Solar Wind Spatial Scales in and Comparisons of Hourly Wind and ACE Plasma and Magnetic Field Data, J. Geophys. Res.,vol. 110, no. A2, p. A02209. doi: 10.1029/2004JA010804.

Koskinen, H. E. J.. and Huttunen, K.E. J., (2006), Geoeffectivity of Coronal Mass Ejections, Space Sci. Rev., 2006, no. 124, p. 169. doi: 10.1007/s11214-006-9103-0.

Longden, N., Denton, M.H., and Honary, F., (2008), Particle Precipitation during ICMEDriven and CIR-Driven Geomagnetic Storms, J. Geophys. Res., vol. 113, p. A06205. doi: 10.1029/2007JA012752. 2008 .

Lopez, R. E., (1987), Solar Cycle Invariance in Solar Wind Proton Temperature Relationships, J. Geophys. Res., vol. 92, p. 11189.

Mustajab, F., Badruddin, (2011), Geoeffectiveness of the interplanetary manifestations of coronal mass ejections and solar-wind streamstream interactions, Astrophys Space Sci (2011) 331: 91104 DOI 10.1007/s10509-010-0428-5

Nikolaeva, N. S., Yu. I. Yermolaev, and I. G. Lodkina, (2011), Dependence of Geomagnetic Activity during Magnetic Storms on the Solar Wind Parameters for Different Types of Streams, Geomagnetism and Aeronomy, Vol. 51, No. 1, pp. 49-65. (Geomagnetizm i Aeronomiya, 2011, Vol. 51, No. 1, pp. 51-67.)

Nikolaeva, N. S., Yu. I. Yermolaev, and I. G. Lodkina, (2012), Dependence of Geomagnetic Activity during Magnetic Storms on the Solar Wind Parameters for Different Types of 
Streams. 2. Main Phase of Storms, Geomagnetism and Aeronomy, in Press

Plotnikov, I. Ya. and Barkova, E.S., (2007), Nonlinear Dependence of Dst and AE Indices on the Electric Field of Magnetic Clouds, Adv. Space Res., vol. 40, p. 1858.

Pulkkinen, T. I., Partamies, N., Huttunen, K. E. J., Reeves, G. D., and Koskinen, H. E. J., (2007a), Differences in geomagnetic storms driven by magnetic clouds and ICME sheath regions, Geophys. Res. Lett., 34, L02105, doi:10.1029/2006GL027775.

Pulkkinen, T. I., Partamies, N., McPherron, R. L., Henderson, M., Reeves, G. D., Thomsen, M. F., and Singer, H. J., (2007b), Comparative statistical analysis of storm time activations and sawtooth events, J. Geophys. Res., 112, A01205, doi:10.1029/2006JA012024.

Rostoker, G. and Falthammar, C.-G., (1967), Relationship between changes in the interplanetary magnetic field and variations in the magnetic field at the earths surface, J. Geophys. Res., 72, pp. 5853-5863.

Russell, C. T., McPherron, R. L., and Burton, R. K., (1974), On the cause of magnetic storms, J. Geophys. Res., 79, 1105-1109.

Tsurutani, B. T., Gonzalez, W. D., Tang, F., Akasofu, S. I., and Smith, E. J., (1988), Origin of Interplanetary Southward Magnetic Fields Responsible for Major Magnetic Storms Near Solar Maximum (1978-1979), J. Geophys. Res., 93(A8), 8519-8531.

Tsurutani, B. T. and Gonzalez, W. D. (1997), The interplanetary Causes of Magnetic Storms: A Review, in: Magnetic Storms, edited by: Tsurutani, B. T., Gonzalez, W. D., and Kamide, Y., Amer. Geophys. Union Press, Washington D.C., Mon. Ser., 98, p. $77,1997$. 
Turner, N. E., Cramer, W.D., Earles, S.K., and Emery, B.A., (2009), Geoefficiency and Energy Partitioning in CIR-Driven and CME-Driven Storms, J. of Atmosph. and Sol.Terrest. Phys., vol. 71, pp. 1023-1031.

Yermolaev, Yu. I. and Yermolaev, M. Yu. (2002), Statistical Relationships between Solar, Interplanetary, and Geomagnetospheric Disturbances, 19762000, Cosmic Res., 40(1), $1-14$.

Yermolaev, Yu. I. and M. Yu. Yermolaev, (2006), Statistic Study on the Geomagnetic Storm Effectiveness of Solar and Interplanetary Events, Adv. Space Res. 37 (6), 11751181.

Yermolaev, Yu. I., Yermolaev, M. Yu., Lodkina, I. G., and Nikolaeva, N. S. (2007a), Statistic study of heliospheric conditions resulting in magnetic storm, Cosmic Res., $45(1), 1-8$.

Yermolaev, Yu. I., Yermolaev, M. Yu., Lodkina, I. G., and Nikolaeva, N. S. (2007b), Statistic study of heliospheric conditions resulting in magnetic storm, 2. Cosmic Res., 45(6), 489-498.

Yermolaev, Yu. I., Yermolaev, M. Yu., Nikolaeva, N. S., and Lodkina, L. G. (2007c), Interplanetary conditions for CIR-induced and MC induced geomagnetic storms, Bulg. J. Phys., 34, 128-135.

Yermolaev, Yu. I., et al., (2009), Catalog of Large-Scale Solar Wind Phenomena during 1976-2000, Kosm. Issled., vol. 47, no. 2, pp. 99-113. [Cosmic Research, pp. 81-94].

Yermolaev, Yu.I., and M.Yu. Yermolaev, (2010), Solar and Interplanetary Sources of Geomagnetic Storms: Space Weather Aspects, Izvestiya, Atmospheric and Oceanic Physics, Vol. 46, No. 7, pp. 799-819. 
Yermolaev, Yu. I., N. S. Nikolaeva, I. G. Lodkina, and M. Yu. Yermolaev, (2010a), Relative Occurrence Rate and Geoeffectiveness of Large-Scale Types of the Solar Wind, Kosmicheskie Issledovaniya, Vol. 48, No. 1, pp. 3-32. (Cos. Res., 2010, Vol. 48, No. 1, pp. 1-30).

Yermolaev, Y. I.; Nikolaeva, N. S.; Lodkina, I. G.; Yermolaev, M. Yu, (2010b), Large-scale solar wind structures: occurrence rate and geoeffectiveness, 12-th International Solar Wind Conference, AIP Conference Proceedings, Volume 1216, pp. 648-651.

Yermolaev, Yu. I., N. S. Nikolaeva, I. G. Lodkina, and M. Yu. Yermolaev, (2010c), Specific interplanetary conditions for CIR-, Sheath-, and ICME-induced geomagnetic storms obtained by double superposed epoch analysis, Ann. Geophys., 28, 2177-2186.

Yermolaev, Yu.I., Lodkina, I.G., Nikolaeva, N.S., and Yermolaev, M.Yu., (2010d), Statistical Study of Interplanetary Condition Effect on Geomagnetic Storms, Kosm.Issled., vol. 48, no. 6, pp. 499-515. [Cosmic Research, pp. 485-501].

Yermolaev, Yu.I., I.G. Lodkina, N.S. Nikolaeva, M.Yu. Yermolaev, (2011), Statistical Study of Interplanetary Condition Effect on Geomagnetic Storms: 2. Variations of Parameters, Cosmic Research, Vol. 49, No. 1, pp. 21-34.

Zhang, J., Richardson, I. G., Webb, D. F., Gopalswamy, N., Huttunen, E., Kasper, J. C., Nitta, N. V., Poomvises, W., Thompson, B. J., Wu, C.-C., Yashiro, S., and Zhukov, A. N. (2007), Solar and interplanetary sources of major geomagnetic storms (Dst $<-100$ nT) during 1996-2005, J. Geophys. Res., 112, A10102, doi:10.1029/2007JA012321. 

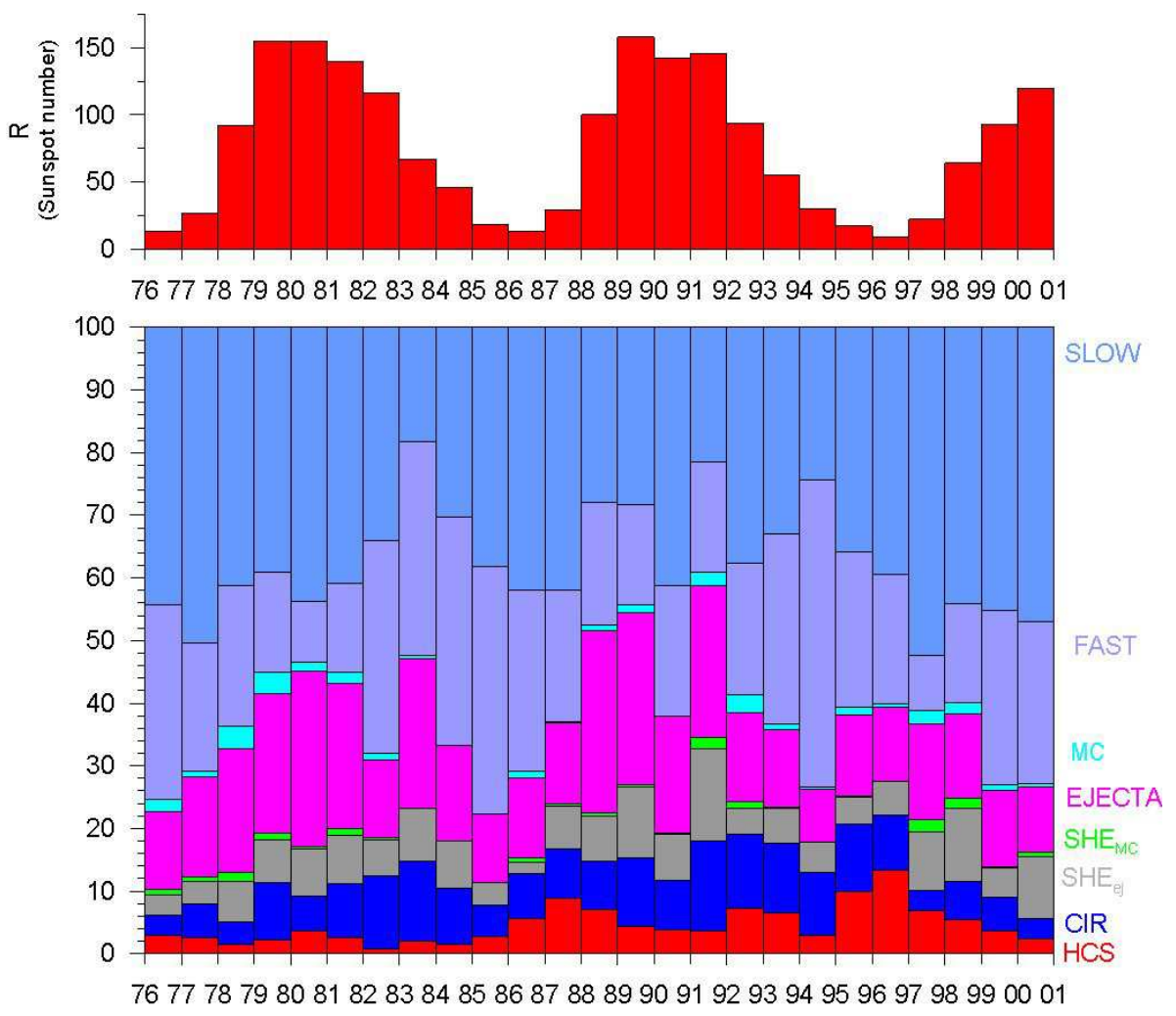

Figure 1. Yearly average values of sunspots (top panel) and yearly average distributions of times of observations for different types of solar wind (\%, bottom panel).
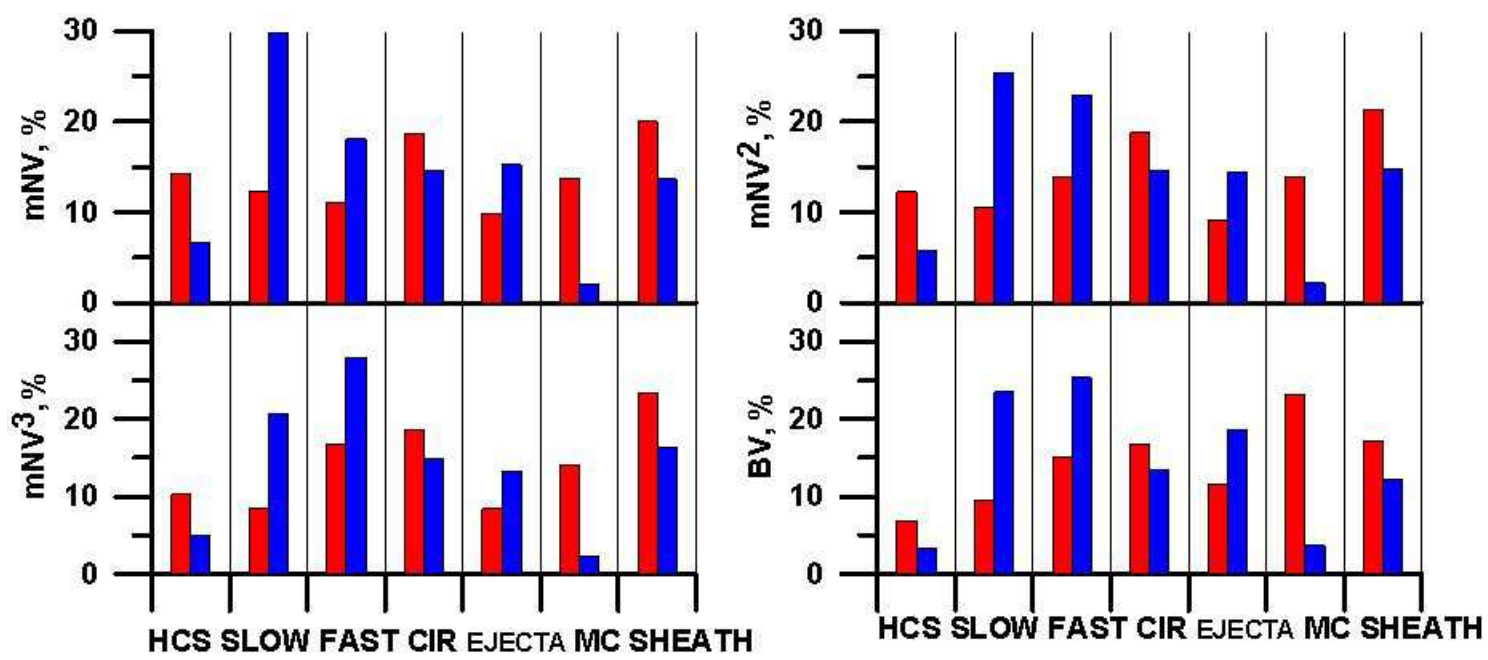

Figure 2. Average values (red columns) and Suns losses (blue columns) mass, momentum, energy and magnetic fluxes for different types of solar wind streams (\%). 


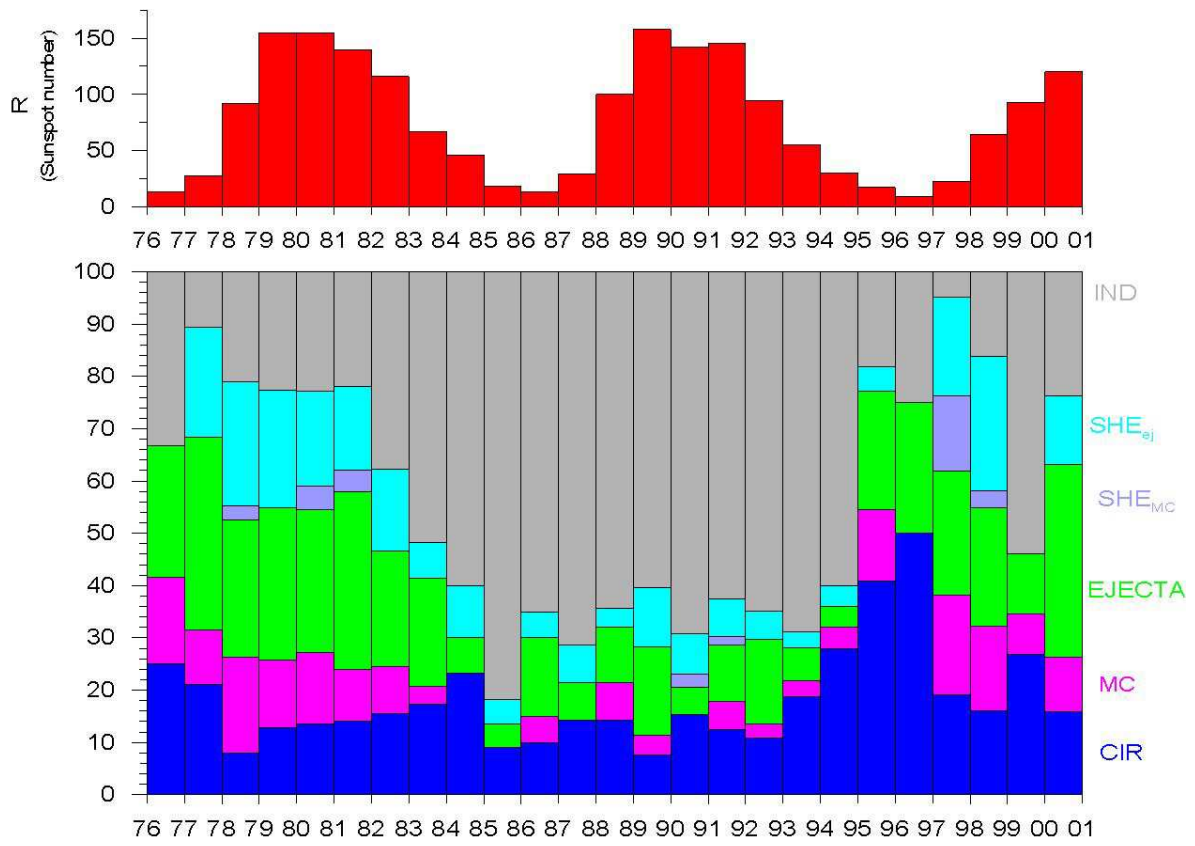

Figure 3. Sunspot number (top panel) and year-averaged distributions of magnetic storms with $D s t<-50$ nT over types of their interplanetary drivers (\%, bottom panel).

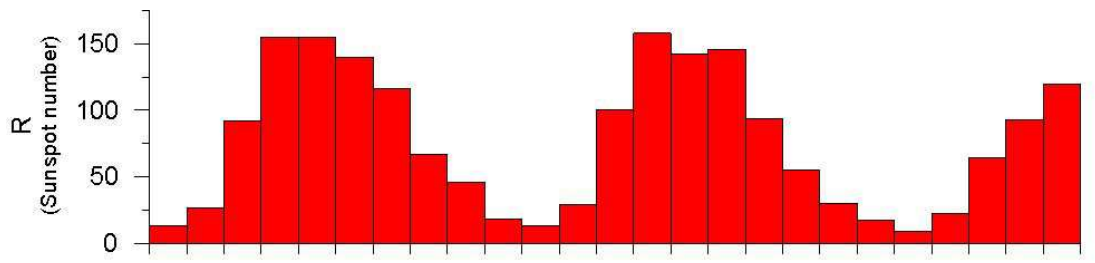

7677787980818283848586878889909192939495969798990001

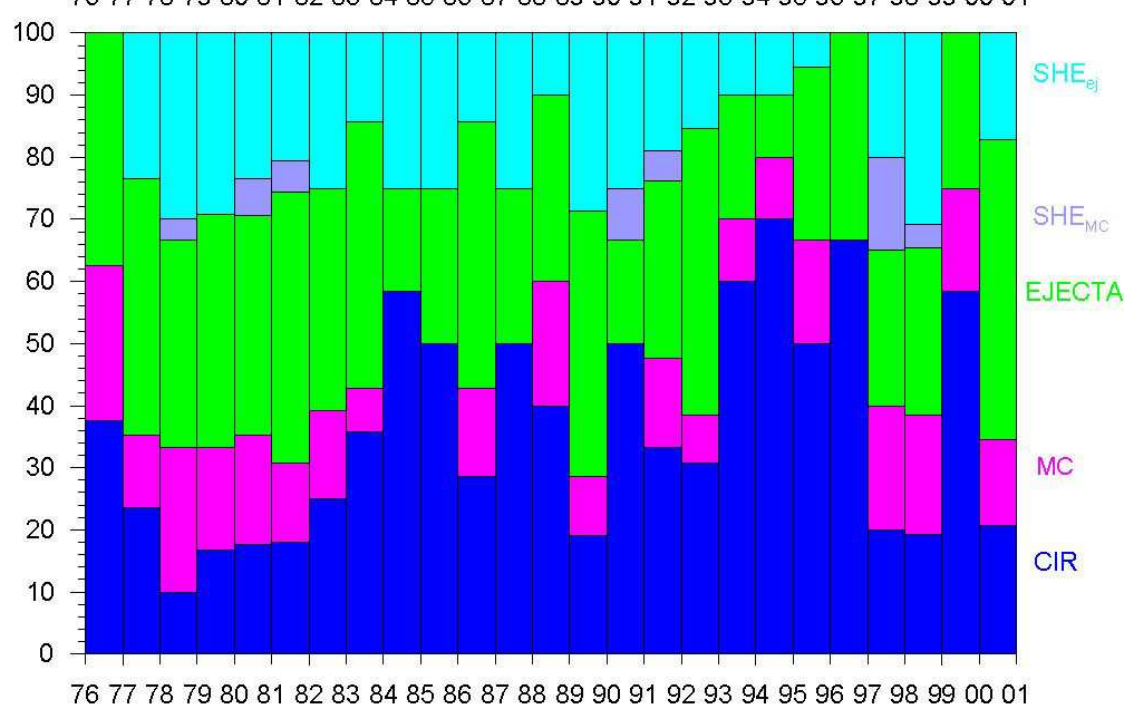

Figure 4. The same as in Figure 3 when IND storms was excluded from analyses 
Table 1. Time observation of different types of solar wind streams during 1976-2000

\begin{tabular}{lc}
\hline Types of solar wind & Time observations, \% \\
\hline Slow & $31 \pm 7$ \\
Fast & $21 \pm 8$ \\
HCS & $6 \pm 4$ \\
CIR & $10 \pm 3$ \\
Ejecta & $20 \pm 6$ \\
MC & $2 \pm 1$ \\
Sheath before Ejecta & $8 \pm 4$ \\
Sheath before MC & $0.8 \pm 0.7$ \\
\hline
\end{tabular}

Table 2. Probability of generation of magnetic storms with $D s t \leq-50 n T$ (geoeffectiveness)

for different types of solar wind streams during 1976-2000

\begin{tabular}{lcccc}
\hline Types of solar wind & $\begin{array}{c}\text { Number of observations } \\
\text { of interplanetary } \\
\text { events }\end{array}$ & $\begin{array}{c}\text { Number of storms } \\
\text { induced by this } \\
\text { type of events }\end{array}$ & $\begin{array}{c}\text { Part from } \\
\text { identified } \\
\text { storms, } \%\end{array}$ & Geoeffectiveness \\
\hline CIR & 717 & 145 & 31.2 & 0.202 \\
Sheath before MC & 79 & 12 & 2.6 & 0.142 \\
Sheath before Ejecta & 543 & 84 & 18.1 & 0.155 \\
MC with Sheath & 79 & 50 & 13.4 & 0.633 \\
MC without Sheath & 22 & 12 & 2.6 & 0.545 \\
Ejecta with Sheath & 543 & 115 & 24.8 & 0.212 \\
Ejecta without Sheath & 585 & 46 & 9.9 & 0.078 \\
\hline
\end{tabular}


Table 3. Ratio of magnetospheric indices to integrated IMF $B z$ and $E y$ fields (at fixed values of $\int_{0}^{t} B z(\tau) d \tau=-30 \mathrm{~h}^{*} \mathrm{nT}$ and $\int_{0}^{t} E y(\tau) d \tau=12 \mathrm{~h} * \mathrm{mV} / \mathrm{m}$ ) ${ }^{\mathrm{a}}$

\begin{tabular}{l|cccc|cccc}
\hline SW type & $D s t / B z$ & $D s t^{*} / B z$ & $K p / B z$ & $A E / B z$ & $D s t / E y$ & $D s t^{*} / E y$ & $K p / E y$ & $A E / E y$ \\
\hline$C I R$ & 2.4 & 2.8 & 0.18 & 22.7 & 5.0 & 6.8 & 0.45 & 56.8 \\
Ejecta & 2.6 & 2.6 & 0.17 & 22.0 & 6.1 & 6.8 & 0.43 & 53.8 \\
$M C$ & 1.9 & 2.1 & 0.17 & 22.3 & 4.3 & 4.9 & 0.42 & 54.2 \\
Ejecta $+M C$ & 2.3 & 2.6 & 0.17 & 21.8 & 5.3 & 6.0 & 0.42 & 53.3 \\
Sheath & 2.4 & 3.0 & 0.20 & 24.3 & 4.9 & 6.3 & 0.46 & 57.9 \\
IND & 2.9 & 2.6 & 0.18 & 24.0 & 6.5 & 6.1 & 0.44 & 48.9 \\
a Dimensions of coefficients: $\left[D s t / B z, D s t^{*} / B z, A E / B z\right]=\mathrm{nT} /\left(\mathrm{h}^{*} \mathrm{nT}\right),[K p / B z]=1 /\left(\mathrm{h}^{*} \mathrm{nT}\right)$,
\end{tabular}

$\left[D s t / E y, D s t^{*} / E y, A E / E y\right]=\mathrm{nT} /\left(\mathrm{h}^{*} \mathrm{mV} / \mathrm{m}\right)$, and $[K p / E y]=1 /\left(\mathrm{h}^{*} \mathrm{mV} / \mathrm{m}\right)$ 